\title{
Pyrotinib in the treatment of advanced lung adenocarcinoma with HER2 exon 20 mutation: a case report and literature review
}

\author{
Ying Luo"^ ${ }^{\#}$ Bin Yao", Ping Huang, Yiting Zhao, Jun Chen \\ Department of Radiotherapy and Chemotherapy, The Affiliated People's Hospital, Ningbo University, Ningbo, China \\ \#These authors contributed equally to this work. \\ Correspondence to: Jun Chen. Department of Radiotherapy and Chemotherapy, The Affiliated People's Hospital, Ningbo University, Ningbo, China. \\ Email: 404868362@qq.com.
}

\begin{abstract}
The lung cancer poses a great threat to the patients' health and social activities. Luckily, epithelial growth factor receptor tyrosine kinase inhibitors (EGFR-TKIs) have greatly improved the response of patients getting EGFR-mutated non-small cell lung cancer (NSCLC), but the targeted therapy of human epidermal growth factor receptor 2 (HER2) mutations in lung cancer is still being explored; however, the effectiveness of drugs for HER2-mutant NSCLC is unsatisfactory. This article reported the diagnosis and treatment of a case of advanced lung adenocarcinoma with erb-b2 receptor tyrosine kinase 2 (ERBB2, also known as HER2) exon 20 mutation. A 65 -year-old women received concurrent chemoradiotherapy postoperatively after a lesion was found in her lung. A new metastatic lesion then appeared in the lung 8 months later and she diagnosed with stage IV lung adenocarcinoma; The gene detection found the patient carried a nonframeshift insertion mutation of HER2 exon 20, According to the test result, the patient received pyrotinib, and the new lesion in the lung disappeared soon. The progression-free survival (PFS) of the patient was up to 26 months. The clinical study reported a such successful application of pyrotinib in a patient with HER2mutated advanced lung adenocarcinoma, the case indicated that pyrotinib may provide a longer survival time for such patients.
\end{abstract}

Keywords: Lung cancer; human epidermal growth factor receptor 2 (HER2); pyrotinib; case report

Submitted Oct 26, 2021. Accepted for publication Dec 20, 2021.

doi: 10.21037/apm-21-3468

View this article at: https://dx.doi.org/10.21037/apm-21-3468

\section{Introduction}

Lung cancer is a common malignant tumor, accounting for more than 230,000 illnesses and 130,000 deaths in the United States in 2021 (1). This results a huge burden on society, and also poses a great threat to the health of individuals. The traditional treatment for non-small cell lung cancer (NSCLC) mainly includes surgery, chemotherapy, and radiotherapy; however, these provide limited benefit to patients (2). The emergence of targeted therapy in the $21^{\text {st }}$ century has changed this pattern. A large number of clinical studies have shown that people with genetic mutations will obtain a longer survival time by taking the corresponding targeted drugs (3). However, anti-human epidermal growth factor receptor 2 (HER2) therapy has offered limited progress for lung carcinoma patients with $H E R 2$ mutations. Statistically, the incidence of HER 2 mutations in lung cancer only accounts for approximately $3 \%$, and is more common in women, Asians, non-smokers, and lung adenocarcinoma pathologies. A safe and effective approach is urgently needed for these patients (4). And activating mutations of HER2 have been observed in $1.7 \%$ of lung adenocarcinomas (5). Pyrotinib is an orally administered irreversible dual panErbB

$\wedge$ ORCID: 0000-0003-2975-6699. 

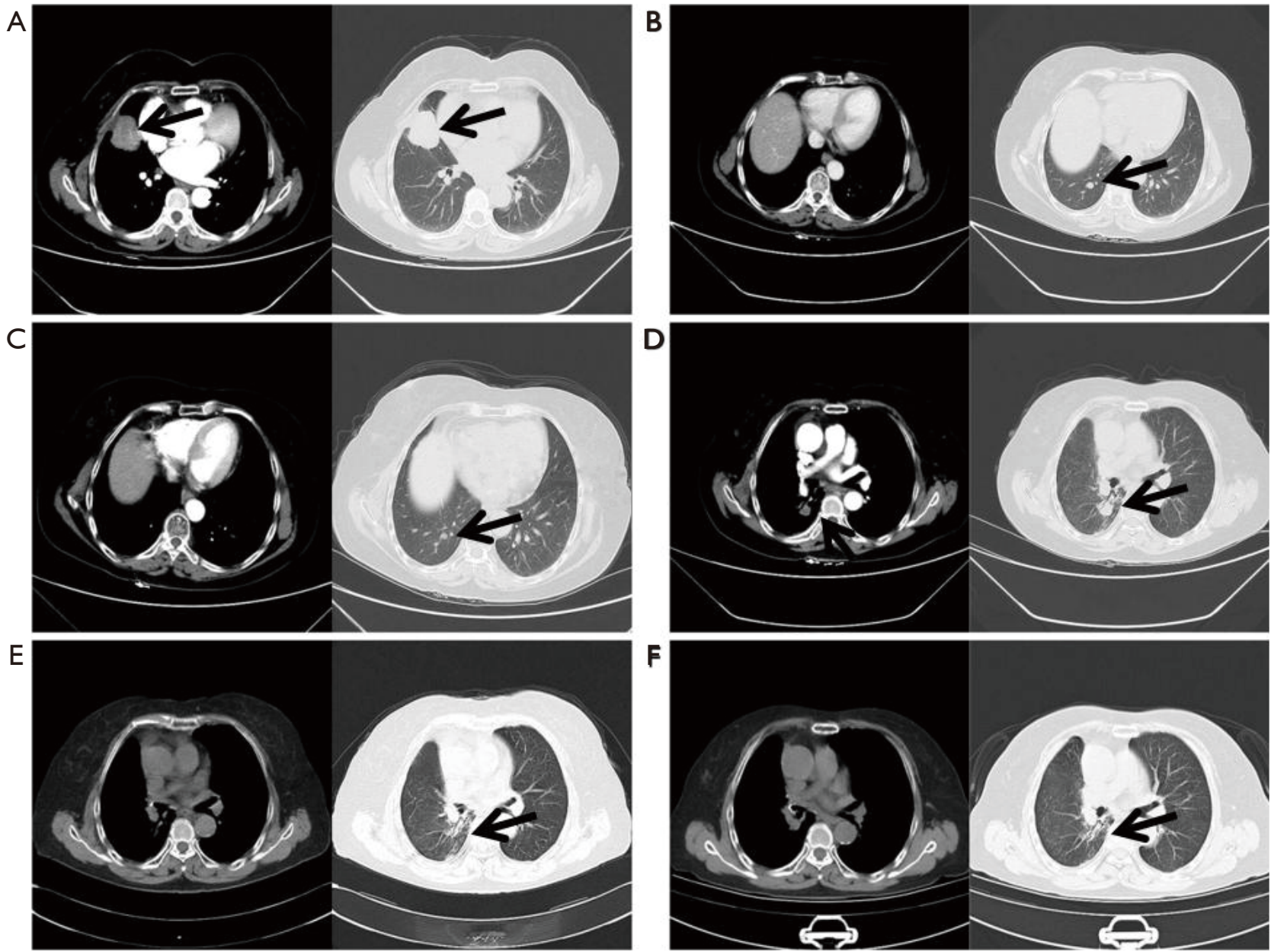

Figure $1 \mathrm{CT}$ scans of the patient before pyrotinib and follow-up after pyrotinib treatment. The meaning of arrows is the location of the lesion. (A) September 27, 2017: physical examination of chest CT, tumor size is $4 \mathrm{~cm} \times 3.5 \mathrm{~cm} \times 3 \mathrm{~cm}$; (B) October 18, 2018: new lesion in the lower right lung; (C) January 27, 2019: CT images after treatment of the right lower lung lesion; (D) April 23, 2019: new lesion in the right hilar; (E) November 28, 2019: CT images after pyrotinib treatment; (F) April 26, 2021: CT images during pyrotinib treatment. CT, computed tomography.

tyrosine kinase inhibitor (TKI) developed by Shanghai Hengrui Pharmaceutical (a subsidiary of Jiangsu Hengrui Medicine) for the treatment of advanced solid tumors with overexpression of HER2 (6). In this report, we share the case of a HER2-mutant advanced lung adenocarcinoma patient treated with pyrotinib for 26 months who achieved stable disease. We present the following article in accordance with the CARE reporting checklist (available at https://dx.doi. org/10.21037/apm-21-3468).

\section{Case presentation}

A mass was found in the right lung of a 65 -year-old female through chest computer tomography (CT) on September 27, 2017 (Figure 1A). She had 2-year history of hypertension, and did not have any specific history of smoking or malignant tumor. A radical resection of the right middle lung lobe and lymph node dissection was performed on October 5, and pathology results indicated invasive adenocarcinoma with $4 \mathrm{~cm} \times 3.5 \mathrm{~cm} \times 3 \mathrm{~cm}$ mass invading the pericardium, as well as one positive lymph node in the third group, visceral pleura, pericardium, and nerves were also invaded. Moreover, a vascular tumor embolism was found. Based on the above pathological results, and combined with the American Joint Committee on Cancer (AJCC) $7^{\text {th }}$ edition guidelines, this patient's 
tumor $(\mathrm{T})$ lymph node $(\mathrm{N})$ metastasis $(\mathrm{M})$ staging was classified as pT3N2M0 (stage IIIA). On October 30, 2017, concurrent chemoradiation was applied [pemetrexed $500 \mathrm{mg} / \mathrm{m}^{2}+$ cisplatin $75 \mathrm{mg} / \mathrm{m}^{2}$, every 3 weeks (q3w), four cycles; radiotherapy dose: $95 \%$ planning target volume $=56 \mathrm{~Gy} / 28 \mathrm{~F}$ ]. On October 18, 2018, a $0.8 \mathrm{~cm}$ solid nodule was discovered in the lower lobe of the right lung (Figure $1 B$ ) and the carcinoembryonic antigen (CEA) had elevated to $12.69 \mathrm{ng} / \mathrm{mL}$. The patient received four cycles of AP (pemetrexed $500 \mathrm{mg} / \mathrm{m}^{2}+$ cisplatin $75 \mathrm{mg} / \mathrm{m}^{2}, \mathrm{q} 3 \mathrm{w}$ ) (Figure 1C), followed by four cycles of $\mathrm{P}$ (pemetrexed $\left.500 \mathrm{mg} / \mathrm{m}^{2}, \mathrm{q} 3 \mathrm{w}\right)$.

On April 23, 2019, another solid nodule appeared on the right hilar (Figure 1D) and the CEA was also elevated to $16.09 \mathrm{ng} / \mathrm{mL}$, but the patient refused chemotherapy. Gene detection was performed via next-generation sequencing using paraffin sections of tissues from the right middle lower mass (Table 1), and the result indicated that the patient carried a non-frameshift insertion mutation of HER2 exon 20 with HER2-p. Tyr772 _Ala775dup, and the frequency was $30.9 \%$. She was diagnosed with stage IV lung adenocarcinoma with a HER2 exon 20 mutation. Pyrotinib (400 mg/qd) was administered and without stopping. At the time of writing this report, the patient's condition was stable (Figure 1E,1F). The patient has experienced one side effect (mild diarrhea). Figure 2 shows the entire treatment process. All procedures performed in this study involving human participants were in accordance with the ethical standards of the institutional and/or national research committee(s) and with the Helsinki Declaration (as revised in 2013). Written informed consent was obtained from the patient for publication of this case report and accompanying images. A copy of the written consent is available for review by the editorial office of this journal.

\section{Discussion}

HER2, a member of the EGFR family, triggers a network of downstream signaling pathways and mediates cell division, adhesion, movement, and death when it binds to the HER 2 receptor, resulting in the occurrence of malignant tumors (7). HER 2 mutations have been detected in approximately $2-4 \%$ of NSCLC patients, $90 \%$ of which are insertions of exon 20 (8). Y772_A775dup mutation accounts for 47.7\% and G778_P780dup mutation accounts for $11.6 \%$ (9). Our patient, an elderly Asian female with HER2-mutant advanced lung adenocarcinoma, carried a common insertion mutation of HER2 exon 20 with
HER2-p. Tyr772 _Ala775dup.

It has been reported that the median duration of response (mDoR) of HER2-mutant metastatic lung cancer patients receiving anti-HER 2 therapy is only 2.2 months (10). Several pan-HER inhibitors, such as afatinib (11), dacomitinib (12), neratinib (13), and poziotinib (14) provide limited efficacy. The fifth edition of the 2021 NCCN guidelines recommends T-DM1 and DS-8201 as targeted therapies for HER2-mutant NSCLC; however, treatment is limited due to the unavailability of drugs. Pyrotinib is a new type of irreversible TKI, which shows a strong anti-tumor effect to inhibit cell proliferation, leading to HER 2 overexpression in vivo and in vitro $(15,16)$. A phase 3 clinical trial (17) found that HER2-mutant metastatic breast cancer patients treated with trastuzumab and taxanes before receiving pyrotinib plus capecitabine had significantly improved progression-free survival (PFS) compared with those receiving lapatinib plus capecitabine (12.5 vs. 6.8 months, respectively). Therefore, pyrotinib has been approved as a second-line treatment for HER2positive metastatic breast cancer in China. It behaves well in breast cancer and also provides positive results in HER2positive lung cancer. In a phase II trial, 15 HER2-mutant advanced NSCLC patients treated with pyrotinib had an objective response rate (ORR) of $53.3 \%$ and a median overall survival (mOS) of 6.4 months (18). In another phase II study (19), patients with stage IIIB and IV HER2mutant lung adenocarcinoma treated with platinum-based chemotherapy prior to being enrolled to receive pyrotinib had a PFS of 6.9 months and a mOS of 14.4 months. In the 2021 ASCO online annual meeting (20), it was reported that the ORR of applying pyrotinib combined with apatinib for HER2-mutant advanced NSCLC reached $45.5 \%$, and the ORR of patients with brain metastases was $46.2 \%$. The above research illustrates the feasibility of pyrotinib for the treatment of HER2-mutant NSCLC.

Moreover, the side effects of pyrotinib are controllable. Treatment-related adverse events (TRAEs) of any grade were reported in 59 patients (98.3\%), with most of these being grades 1 or 2 . The most common TRAEs were diarrhea $(91.7 \%)$, elevated blood creatinine $(30.0 \%)$, vomiting $(28.3 \%)$, elevated alanine aminotransferase $(15.0 \%)$, and elevated aspartate aminotransferase (15.0\%). Grades 3-4 TRAEs occurred in 17 patients (28.8\%), and the most common TRAE was diarrhea (17), which usually occurred on the fourth day and remained for 2-3 days after taking pyrotinib. Symptomatic treatment or reducing the dose of pyrotinib was found to relieve symptoms. For our 
Table 1 Genes in April 2019

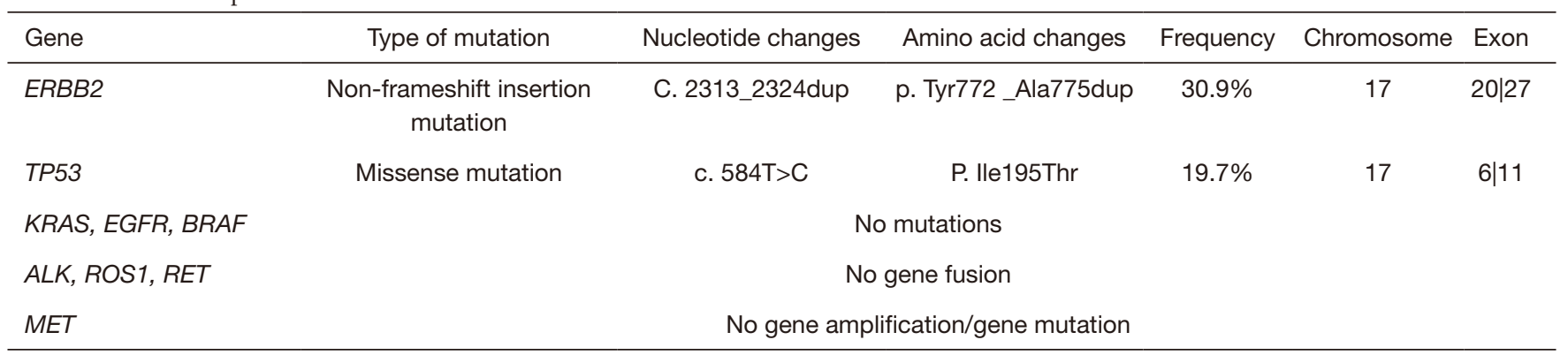

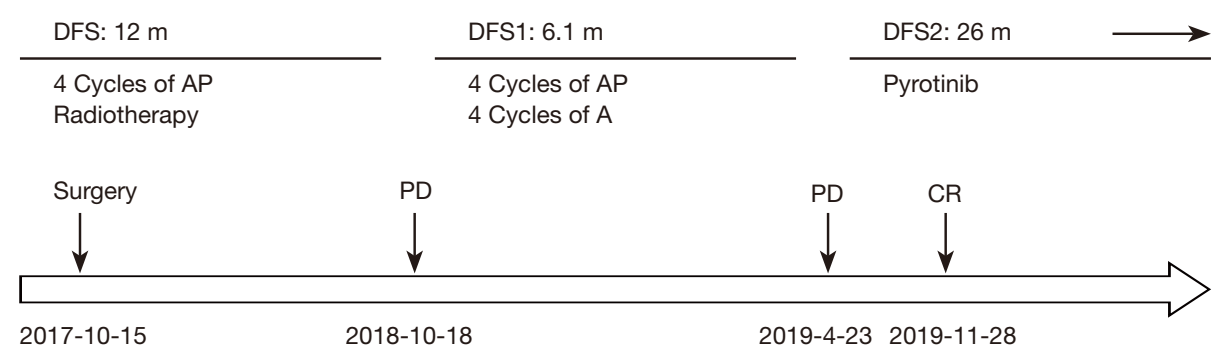

Figure 2 The entire treatment process. DFS, disease-free survival; AP, pemetrexed and cisplatin; PD, progressive disease; CR, complete response.

patient, the side effect was mild diarrhea, and no grade III or IV adverse reactions appeared. Due to the accessibility and benefits of pyrotinib, we applied the new novel generation of targeted drug to the patient. The patient's condition has been stable for over 26 months with mild side effects, and we will continue focus on the patient's treatment. We make a suggestion that pyrotinib might be a potential treatment for patients with HER2-positive advanced adenocarcinoma because of the favorable response and clinical benefit.

However, there are some limitations in this study that should be noted. Firstly, although pyrotinib has provided long-term benefits to this patient, the mechanism of pyrotinib resistance and the reason behind HER2 resistance mutations can be explored if the patient becomes resistant to pyrotinib in the future. Secondly, there are few clinical research statistics on III HER2-mutant NSCLC, and further studies should be conducted to demonstrate the efficacy and safety of pyrotinib.

\section{Conclusions}

Pyrotinib may provide longer-term survival for HER2 mutation-positive NSCLC, and needs to be further applied clinically.

\section{Acknowledgments}

Funding: This work was supported by Ningbo Science and Technology Bureau of Zhejiang Province (2021S116).

\section{Footnote}

Reporting Checklist: The authors have completed the CARE reporting checklist. Available at https://dx.doi. org/10.21037/apm-21-3468

Conflicts of Interest: All authors have completed the ICMJE uniform disclosure form (available at https://dx.doi. org/10.21037/apm-21-3468). The authors have no conflicts of interest to declare.

Ethical Statement: The authors are accountable for all aspects of the work in ensuring that questions related to the accuracy or integrity of any part of the work are appropriately investigated and resolved. All procedures performed in this study involving human participants were in accordance with the ethical standards of the institutional and/or national research committee(s) and with the Helsinki Declaration (as revised in 2013). Written informed consent was obtained from the patient for publication of this case report and 
accompanying images. A copy of the written consent is available for review by the editorial office of this journal.

Open Access Statement: This is an Open Access article distributed in accordance with the Creative Commons Attribution-NonCommercial-NoDerivs 4.0 International License (CC BY-NC-ND 4.0), which permits the noncommercial replication and distribution of the article with the strict proviso that no changes or edits are made and the original work is properly cited (including links to both the formal publication through the relevant DOI and the license). See: https://creativecommons.org/licenses/by-nc-nd/4.0/.

\section{References}

1. Siegel RL, Miller KD, Fuchs HE, et al. Cancer Statistics, 2021. CA Cancer J Clin 2021;71:7-33.

2. Duma N, Santana-Davila R, Molina JR. Non-Small Cell Lung Cancer: Epidemiology, Screening, Diagnosis, and Treatment. Mayo Clin Proc 2019;94:1623-40.

3. Imyanitov EN, Iyevleva AG, Levchenko EV. Molecular testing and targeted therapy for non-small cell lung cancer: Current status and perspectives. Crit Rev Oncol Hematol 2021;157:103194.

4. Shigematsu H, Takahashi T, Nomura M, et al. Somatic mutations of the HER2 kinase domain in lung adenocarcinomas. Cancer Res 2005;65:1642-6.

5. Cancer Genome Atlas Research Network. Comprehensive molecular profiling of lung adenocarcinoma. Nature 2014;511:543-50.

6. Blair HA. Pyrotinib: First Global Approval. Drugs 2018;78:1751-5.

7. Yarden Y, Sliwkowski MX. Untangling the ErbB signalling network. Nat Rev Mol Cell Biol 2001;2:127-37.

8. Koga T, Kobayashi Y, Tomizawa K, et al. Activity of a novel HER2 inhibitor, poziotinib, for HER2 exon 20 mutations in lung cancer and mechanism of acquired resistance: An in vitro study. Lung Cancer 2018;126:72-9.

9. Zhao S, Fang W, Pan H, et al. Conformational Landscapes of HER2 Exon 20 Insertions Explain Their Sensitivity to Kinase Inhibitors in Lung Adenocarcinoma. J Thorac Oncol 2020;15:962-72.

10. Eng J, Hsu M, Chaft JE, et al. Outcomes of chemotherapies and HER2 directed therapies in advanced HER2-mutant lung cancers. Lung Cancer 2016;99:53-6.

11. Lai WV, Lebas L, Barnes TA, et al. Afatinib in patients with metastatic or recurrent HER2-mutant lung cancers: a retrospective international multicentre study. Eur J Cancer
2019;109:28-35.

12. Kris MG, Camidge DR, Giaccone G, et al. Targeting HER2 aberrations as actionable drivers in lung cancers: phase II trial of the pan-HER tyrosine kinase inhibitor dacomitinib in patients with HER2-mutant or amplified tumors. Ann Oncol 2015;26:1421-7.

13. Hyman DM, Piha-Paul SA, Won H, et al. HER kinase inhibition in patients with HER2- and HER3-mutant cancers. Nature 2018;554:189-94.

14. Robichaux JP, Elamin YY, Tan Z, et al. Mechanisms and clinical activity of an EGFR and HER2 exon 20-selective kinase inhibitor in non-small cell lung cancer. Nat Med 2018;24:638-46.

15. Zhu Y, Li L, Zhang G, et al. Metabolic characterization of pyrotinib in humans by ultra-performance liquid chromatography/quadrupole time-of-flight mass spectrometry. J Chromatogr B Analyt Technol Biomed Life Sci 2016;1033-1034:117-27.

16. Li X, Yang C, Wan H, et al. Discovery and development of pyrotinib: A novel irreversible EGFR/HER2 dual tyrosine kinase inhibitor with favorable safety profiles for the treatment of breast cancer. Eur J Pharm Sci 2017;110:51-61.

17. Xu B, Yan M, Ma F, et al. Pyrotinib plus capecitabine versus lapatinib plus capecitabine for the treatment of HER2-positive metastatic breast cancer (PHOEBE): a multicentre, open-label, randomised, controlled, phase 3 trial. Lancet Oncol 2021;22:351-60.

18. Wang Y, Jiang T, Qin Z, et al. HER2 exon 20 insertions in non-small-cell lung cancer are sensitive to the irreversible pan-HER receptor tyrosine kinase inhibitor pyrotinib. Ann Oncol 2019;30:447-55.

19. Zhou C, Li X, Wang Q, et al. Pyrotinib in HER2-Mutant Advanced Lung Adenocarcinoma After Platinum-Based Chemotherapy: A Multicenter, Open-Label, Single-Arm, Phase II Study. J Clin Oncol 2020;38:2753-61.

20. Yang G, Xu H, Yang L, et al. Efficacy and safety of pan-ErbB inhibitor pyrotinib combined with antiangiogenic agent apatinib for HER2-mutant or amplified metastatic NSCLC: A phase II clinical study. J Clin Oncol 2021;39:abstr 9035.

(English Language Editor: A. Kassem)

Cite this article as: Luo Y, Yao B, Huang P, Zhao Y, Chen J. Pyrotinib in the treatment of advanced lung adenocarcinoma with HER2 exon 20 mutation: a case report and literature review. Ann Palliat Med 2021;10(12):12906-12910. doi: 10.21037/ apm-21-3468 